near Geneva (see page 174). De Rose, then France's representative to the United Nations Atomic Energy Commission, chaired the meeting. He had got caught up in the process after becoming friends with Robert Oppenheimer, one of CERN's earliest proponents. De Rose said in a separate interview with Nature that CERN was the result of the capacity of scientists such as Oppenheimer to propose grand ideas, and worry about obstacles later.

Although this approach does not always work, the next few weeks will show that it really has changed the world. In the ensuing half-century, CERN has revolutionized our understanding of the subatomic world; with the switching-on this week of the Large Hadron Collider (see page 156) it promises to scale new heights.

When we began to think about commissioning this series, several difficulties arose. First, we were looking for more than the traditional scientific conference, and it was notable how few of the twentieth century's world-changing meetings had involved scientists taking a lead. As a list emerged, we were faced with another problem: time had sadly depleted the pool of writers. This week's author, for example, is among the few surviving members of a group that met 57 years ago.

The six events that made the final cut took place on three continents and span five decades, from 1951 to the dawn of the new millennium. They represent the twentieth century's promise, and two of its greatest threats. And they illustrate a period in history when scientists felt they should raise a collective voice to advance the public good. The six meetings have something else in common. In wanting to change their world, the scientists involved needed and obtained the support of governments and, in some cases, the media.

In two of the conferences - those related to CERN and the Human Genome Project - scientists organized themselves and others to create new and exciting research endeavours. But the other meetings considered in our series had very different aims. At a 1975 conference held in Asilomar, California, for example, geneticists felt compelled to sound an alarm over DNA modification, then a new technology of uncertain impact. At a meeting in Bellagio, Italy, in 1969, plant scientists were among those who convinced governments and philanthropic foundations to invest in technologies to take the green revolution to the developing world.

As in any series of this nature, some caveats are in order. First, global initiatives are a process in which many decisions are made over many years. In the case of CERN, the Paris 1951 event was not the first official meeting in the institution's history. It was, however, an occasion where private disagreements between governments became public, and where a consensus was eventually found to move the project forward. Without such a consensus, it is debatable whether CERN would have taken the direction it did.

Second, our list is not the final word. There are other candidates for the title of Meetings that Changed the World. And our illustrious attendees' opinions are, of course, personal and often provocative. Readers are invited to have their say at http://network.nature.com/ forums/naturenewsandopinion/2359.

\section{A bigger picture}

\section{Beneath cancer's daunting complexity lies a simplicity that gives grounds for hope.}

-

or several years now, large-scale cancer-genome studies have made it increasingly clear that a tumour cell is a genetic disaster area littered with mutations that differ not only from one type of cancer to the next, but from one patient to the next. Pharmaceutical companies have had to accept that Gleevec, a drug that treats a form of leukaemia by targeting a specific gene product, is almost certainly going to be a rare exception in the therapeutic arsenal; most cancers are far too complex to yield to such a magic bullet.

That message was hammered home with new statistical power in three studies released last week (see page 148). Two of the studies, published in Science by a team based at Johns Hopkins Kimmel Cancer Center in Baltimore, Maryland, focused on pancreatic cancer and a type of brain cancer called glioblastoma multiforme - both among the most fatal and intractable tumours known. The third paper, published in Nature by the Cancer Genome Atlas project, also focused on glioblastoma. The studies took a more comprehensive approach than previous large cancer-genomics studies, by simultaneously analysing genetic sequences, copy-number variations, expression arrays and other forms of data. The Johns Hopkins team looked at all the active genes in tumours from a few dozen patients; the Genome Atlas team looked at selected genes in tumours from 206 patients. Taken together, their results show that no single mutated gene lies at the heart of any of these tumours. The pancreatic tumour samples, for example, showed an average of 63 genetic mutations each - with considerable variation from one sample to the next.

That conclusion might make the prospects for new targeted drug therapies for cancer seem hopeless. And yet, the reality may be just the opposite. The richness of the data becoming available in these and other studies allows researchers to cut through the complexity. Genes work together in pathways of reactions to accomplish a particular biological function, such as cell division - and many or most of the mutated genes picked up by these cancer studies are involved in a comparatively small number of pathways. The Johns Hopkins team found that most of the mutations in their pancreatic tumours affected just 12 pathways. The Genome Atlas team found that most of its glioblastomas showed mutations in a set of three pathways. So drugs targeting these pathways might work in more patients than drugs that target only one of a pathway's myriad gene components.

To realize that hope, researchers and funding agencies will need to do many more such studies on many more types of cancer. Just as important is the next step, which is to determine how these mutated pathways contribute to the development of cancer - and how that contribution might be removed. After that comes the task of finding useful biomarkers, chemical signals that will allow therapists to determine which pathways have been affected in each cancer patient, and how that patient will respond to any given therapy.

None of this will be easy. Untangling the immense complexity of cancer will be big science by anyone's definition, requiring a longterm commitment and enormous amounts of data. And yet, that very complexity has begun to give reason for optimism. 